\title{
Editorial
}

\section{Discontinuing Antidepressant Drugs: Lesson from a Failed Trial and Extensive Clinical Experience}

\author{
Giovanni A. Fava ${ }^{a-c}$ Carlotta Belaise ${ }^{a, c}$ \\ ${ }^{a}$ Department of Psychology, University of Bologna, Bologna, Italy; ${ }^{b}$ Department of Psychiatry, State University of \\ New York at Buffalo, Buffalo, NY, USA; ' Affective Disorders Program, Analisi Mediche Pavanello, Padova, Italy
}

Scholten et al. [1] reported on the first randomized controlled trial that attempted to prevent relapse in patients with remitted anxiety disorder who discontinued antidepressant drugs (AD) by use of a cognitive-behavioral therapy (CBT) relapse prevention group compared to treatment as usual. The patients who were assigned to CBT received 8 group sessions of relapse prevention, targeting vulnerability factors and discontinuation symptoms. AD were tapered every 2 weeks within 4 months according to a fixed schedule. In the control group (treatment as usual), tapering and discontinuation of $\mathrm{AD}$ were carried out without CBT, in individual sessions, according to the same schedule [1]. Primary outcomes were occurrence/reoccurrence of any anxiety disorder or major depressive disorder. Secondary outcome was the success rate of discontinuation of AD. Seventy-three patients were enrolled. Over 16 months there were no significant differences between the CBT group and the treatment as usual group in any of the primary and secondary outcome measures. Despite guidance, only $36 \%$ of all participants succeeded in discontinuing $\mathrm{AD}$, and only $28 \%$ did not have recurrence. One patient committed suicide. The tri- al was stopped prematurely for ethical reasons and futility [1]. However, it was certainly not futile and provided important insights into discontinuing $\mathrm{AD}$.

As the authors commented [1], the guidelines' recommendations to discontinue $\mathrm{AD}$ were found neither feasible nor effective in their sample. The investigators, while convinced to apply the best evidence, found out that they had been simply misguided. Withdrawal symptoms and syndromes may occur during and despite slow tapering, do not magically vanish after a couple of weeks from discontinuation and may persist for a long time, leading to postwithdrawal syndromes [2,3]. In this trial [1], the CBT group was thus stopped when it was more needed, as we are going to discuss below. Further, withdrawal symptoms and syndromes were not adequately assessed and addressed; they might have been misidentified as the occurrence of an anxiety disorder. That specific trial was bound to fail, but alternative routes may be feasible. We will describe the approach to discontinuing $\mathrm{AD}$ that we have developed over the years. It has not been tested in controlled trials but may inspire new research efforts in an area that badly needs investigation.

\section{KARGER}

(C) 2018 S. Karger AG, Basel

E-Mail karger@karger.com

www.karger.com/pps
Giovanni A. Fava, MD

Department of Psychology, University of Bologna

Viale Berti Pichat 5

IT-40127 Bologna (Italy)

E-Mail giovanniandrea.fava@unibo.it 


\section{Gaining Insights from Extensive Clinical and Research Experience}

In the nineties, the first author (G.A.F.), a psychiatrist, designed and conducted 2 randomized controlled trials concerned with the sequential model of treatment of depression: pharmacotherapy of the acute episode followed by psychotherapy or clinical management while $\mathrm{AD}$ drugs were tapered and discontinued $[4,5]$. He personally treated all 88 patients who entered the trials; only in 8 cases was tapering stopped due to re-emergence of depressed mood (in 6 tapering was successful a few months later outside of these studies; unpubl. findings). The drugs that were used were mostly tricyclic antidepressants. In no cases were withdrawal symptoms observed. It appeared to be pretty clear in those days that antidepressant drugs were not likely to induce dependence and withdrawal symptoms upon tapering and discontinuation. It was different with benzodiazepines (BZD), as demonstrated by another study conducted by the same author in that period. Twenty patients with panic disorder and agoraphobia who had been successfully treated with a standardized behavioral protocol and who were taking BZD had their medications gradually tapered and discontinued [6]. Successful discontinuation was achieved in 16 patients, and 13 suffered from withdrawal symptoms. Discontinuation was not feasible in 4 patients.

In those years, quite a different picture emerged with the introduction of selective serotonin reuptake inhibitors (SSRI). We started being confronted with the withdrawal syndromes that ensued with their discontinuation [7]. A design similar to the one that was employed with BZD [6] was selected for testing the feasibility of getting off SSRI in patients with panic disorder and agoraphobia. Again the first author treated all patients. Twenty subjects who had been successfully treated with a standardized behavioral protocol and who were taking SSRI had their medications gradually tapered and discontinued [8]. We thought we had the best possible conditions for getting SSRI off: patients were panic free after a type of psychotherapy that was associated with enduring effects [9], and they received individual attention with opportunities for clarification and discussion of any symptoms that might have appeared. Yet the results were disappointing. Nine of the patients (45\%) experienced a withdrawal syndrome, which subsided within a month in all but 3 patients who had been taking paroxetine [8]. These 3 patients developed cyclothymia, which they had never experienced before, and presented with a syndrome that was later defined as persistent postwithdrawal disorder
[2], that is the return of original symptoms at greater intensity and/or with additional symptoms related to an emerging new mental disorder. They were all treated with clonazepam: in 1 case the response was good; in another it was very limited, and symptoms subsided when paroxetine was administered again; in the third, clonazepam yielded no improvement, and the symptoms went on for 3 years before fading (he refused taking paroxetine again). Nothing like this had happened with BZD; indeed, anxiety improved upon discontinuation [6], in line with the published literature [10].

The second author (C.B.), a psychologist, gained a lot of experience in treating cases in our Affective Disorders Program that involved withdrawal or postwithdrawal syndromes with SSRI and serotonin-noradrenalin reuptake inhibitors (SNRI) and elaborated the idea that specific psychotherapeutic approaches needed to be developed for overcoming these problems. She did explore the websites of patient associations and published the first report on the wide range of symptomatology that characterized these patients [11]. In a subsequent paper, she outlined a treatment protocol we developed, based on the sequential administration of different psychotherapeutic approaches in patients with persistent postwithdrawal disorders [12].

Another source of insight came from considering withdrawal syndromes together with other clinical phenomena related to AD: the return of depressive symptoms during long-term maintenance; lack of response to a previously effective treatment when it is started again after a drug-free period; switch to hypomania and bipolar course [13]. Such phenomena may overlap in the same patient, for instance the simultaneous onset of withdrawal symptoms and mania upon venlafaxine discontinuation [14].

The clinical manifestations that have been described may be unified under the oppositional model of tolerance [13-16]. According to this model, continued drug treatment may recruit processes that oppose the initial acute effects of a drug. This may explain loss of efficacy. It may also propel the illness to a more malignant and treatmentunresponsive course, as with a bipolar course or paradoxical reactions. When drug treatment ends, oppositional processes may operate for some time, resulting in appearance of withdrawal symptoms, increased vulnerability to relapse, or resistance if treatment is reinstituted.

An additional source of insight came from a systematic review the authors performed on SSRI discontinuation [3]. The conclusion was that SSRI may cause dependence and clinicians need to add SSRI to the list of drugs 
potentially inducing withdrawal symptoms upon discontinuation, together with BZD, barbiturates, and other psychotropic drugs. The term "discontinuation syndrome" that is currently used minimizes the potential vulnerabilities induced by SSRI and has to be replaced by "withdrawal syndrome" [3]. Similar conclusions were reached from a systematic review of SNRI discontinuation [17].

\section{The Setting for Guided AD Discontinuation}

The Affective Disorders Program where we accumulated our clinical experience follows an innovative model of a mental health clinic, which is based on the joint activities of a psychiatrist (with adequate background both in psychopharmacology and psychotherapy), an internist (with psychosomatic background and familiarity with psychiatric problems), and 4 clinical psychologists (providing psychotherapy after the initial evaluation of the psychiatrist) [18]. Its functioning emphasizes a close coordination of the team members, with repeated assessments and sequential combination of treatments [18]. We argue that $\mathrm{AD}$ discontinuation that is performed without medical consultation and adequate psychotherapeutic support entails substantial risks for the patient and is often bound to fail, as was found to be the case in the trial that we discussed at the beginning of this paper [1]. We will outline the complexity and multidisciplinary requirements of the procedure in the following sections.

\section{The Decision of Discontinuing AD}

There are specific circumstances, which are seldom discussed, that may determine the need for discontinuing a specific $\mathrm{AD}$ (the list is not by any means exhaustive):

\section{Medical Reasons}

Long-term treatment with newer-generation AD may cause important side effects (e.g., gastrointestinal symptoms, weight gain, cardiovascular problems, sexual dysfunction, bleeding) [19-21], which may mandate discontinuation and close medical monitoring. For instance, a very common medical reason is gastric symptomatology associated with the use of SSRI and SNRI [19], which may require proton pump inhibitors. Discontinuation of $\mathrm{AD}$ may provide relief of gastric symptoms, yet it may be difficult to decide when to stop proton pump inhibitors. A prolonged use of these latter drugs may increase the level of gastric recovery, and yet it may be associated with the risk of major depressive disorder [22]. Another example is provided by the hypertensive properties of venlafaxine [19]. We observed 3 cases of resistant hypertension, with poor control of blood pressure, despite the use of multiple antihypertensive drugs. Blood pressure control was regained after discontinuation of venlafaxine, and in all 3 cases antihypertensive therapy needed to be titrated to avoid hypotension.

\section{Pregnancy and Breast-Feeding}

There is an increasing literature on the potential harmful effects of $\mathrm{AD}$ during pregnancy; $\mathrm{AD}$ are transmitted to the child during pregnancy and lactation [19]. The decision of interrupting AD should of course be weighed against the risks of depression during pregnancy. A close coordination with the gynecologist is of paramount importance.

\section{Paradoxical Effects}

Switching into hypomania or mania, apathy, onset of suicidality, and deepening of depressed mood may suggest the opportunity of discontinuing $\operatorname{AD}[13,15]$. Fux et al. [23] observed the emergence of depressive symptoms in 7 of 80 patients (9\%) during treatment of panic disorder by fluvoxamine. These patients had no history of mood disorder, and no symptoms of depression were present before the treatment with fluvoxamine. Depressive symptoms abated when fluvoxamine was discontinued and tricyclic antidepressants or clonazepam were prescribed, and reappeared when fluoxetine was administered.

\section{Lack or Loss of Efficacy}

A specific AD may not be effective from the very beginning or may lose its effectiveness after a certain period [24].

\section{Unclear Reasons for Initial Prescription and Automatic Prolongation of Treatment}

$\mathrm{AD}$ are often prescribed without clear psychiatric indications, when the subject is demoralized and/or under stress, and their use may be prolonged for years [25]. In such cases the clinician and the patient may wish to verify the usefulness of this treatment prolongation.

\section{Planned Discontinuation}

In the sequential model of depression, patients who had responded to drug treatment are offered psychotherapy, while $\mathrm{AD}$ are tapered and discontinued $[4,5]$. This 
design was subsequently used in a number of randomized controlled trials and was found to entail significant benefits [26]. It is an intensive, 2-stage approach, where one type of treatment (psychotherapy) is employed to improve symptoms which another type of treatment (pharmacotherapy) was unable to affect. The sequential design is different from maintenance strategies for prolonging clinical responses that therapies of the acute episodes have obtained, as well as from augmentation or switching strategies because of lack of response to the first line of treatment [26].

\section{Improved Clinical Conditions}

A patient who takes $\mathrm{AD}$ for a mood or anxiety disorder may get better with the addition of psychotherapy, as was found to be the case in the study where SSRI were discontinued after behavioral treatment of panic disorder [8].

\section{Patient Preference}

Even though it is listed at the end, it is an issue that is of primary importance, particularly in a setting characterized by shared decision. If a patient wishes to have his/ her $\mathrm{AD}$ tapered and discontinued and the request appears to be reasonable, it is certainly preferable to have the clinician monitoring the process, rather than letting the patient doing it by himself/herself.

\section{The Role of Assessment in Planning Drug Discontinuation}

There have been several reviews on the management of antidepressant discontinuation symptoms, such as the ones by Lejoyeux and Ades [27], Haddad [28], Schatzberg et al. [29], Warner et al. [30], and Wilson and Lader [31]. In these papers, recommendations about treatment discontinuation apply to the "average" patient and are rather general. However, planning drug discontinuation is a highly individualized process, which needs to incorporate variables such as type and duration of $\mathrm{AD}$ treatment, patterns of symptoms, stage of illness, comorbid conditions, timing of phenomena, responses to previous attempts to discontinue, and other clinical distinctions that demarcate major prognostic and therapeutic differences among patients who otherwise seem to be deceptively similar since they share the same diagnosis and the same drug treatment. Such variables should be filtered by clinical judgment $[32,33]$.

There should be clear definitions of "relapse/recurrence," "new withdrawal symptoms," "rebound," and "persistent postwithdrawal disorder," according to the criteria developed by Chouinard and Chouinard [2]: relapse and recurrence are the gradual return of the original symptoms at the same intensity as before treatment, entailing a return of the same episode and a new episode of illness, respectively; new withdrawal symptoms are classic withdrawal symptoms that are new and not part of the patient's original illness; rebound symptoms are a rapid return of the patient's original symptoms at a greater intensity than before treatment; persistent postwithdrawal disorder consists of the return of the original illness at a greater intensity and/or with additional features of the illness, and/or symptoms related to emerging new disorders, with persistence of symptoms for at least 6 weeks after drug withdrawal [2]. The clinician should be familiar with and investigate withdrawal symptoms that appear with SSRI and SNRI discontinuation [2, 3, 17]. Clinimetric indices such as Discontinuation-Emergent Signs and Symptoms [34] may be very helpful. A semistructured research interview for applying Chouinard's diagnostic criteria has also been developed [35].

Withdrawal symptoms can easily be misinterpreted as signs of relapse. Indeed, trial designs that assess the effects of discontinuing AD for inferring efficacy (i.e., a significant increase in depressive symptoms in the patients whose $\mathrm{AD}$ are discontinued and switched to placebo compared to those who continue with treatment) are flawed by lack of consideration and proper assessment of withdrawal events. The clinical difficulty is increased by the fact that relapse and withdrawal syndromes may coexist. Discriminating issues may be as follows:

(a) It is important to have a history of illness progress in the individual patients, since prodromal symptoms of relapse tend to mirror those of the initial episode both in mood [4] and anxiety [9] disorders. Withdrawal symptoms are new symptoms that were not part of the patient's symptomatology [2, 3, 34-36].

(b) In depression, withdrawal symptoms are likely to have an early onset, while recurrent symptoms generally present with a gradual return; this is only a general tendency, however, and not a rule; in anxiety disorders these patterns do not apply.

(c) Withdrawal symptoms have a tendency to wane with time (unless they develop into persistent withdrawal disorders), whereas the opposite trend occurs with prodromal symptoms of relapse $[4,9]$.

Another helpful assessment strategy is macroanalysis $[32,33]$. The method establishes a relationship between co-occurring syndromes and problems on the basis of where treatment should begin in the first place. Macro- 
analysis starts from the assumption that, in most cases, there are functional relationships among problem areas and that the targets of treatment may vary during the course of disturbances. It may include the various manifestations of tolerance we described (the return of depressive symptoms during long-term maintenance; lack of response to a previously effective treatment when it is started again after a drug-free period; paradoxical effects; switch to hypomania and bipolar course) [13]. Such manifestations may be subsumed under the rubric of "iatrogenic comorbidity" that refers to unfavorable modifications in the course, characteristics, and responsiveness of an illness that may be related to treatments previously administered [37]. When medical comorbidity is present, assessment by an internist or other medical specialist is essential. An important prerequisite before discontinuation is in fact that the patient is medically stable. Bainum et al. [38] reported on the severe consequences of abrupt $\mathrm{AD}$ discontinuation, such as delirium, agitation, and irritability, in patients who are critically ill.

\section{Pharmacological Strategies}

There is consensus in the literature [27-30], as summarized by Wilson and Lader [31], that AD should be tapered as slowly as possible, over at least 4 weeks or longer, and the same AD should be reinstated if withdrawal symptoms occur. Another suggested procedure is to switch to fluoxetine, which is less likely to induce discontinuation problems [34]. Such recommendations ignore the complexity of individual clinical situations. It is wishful thinking to believe that there is a single protocol that can be applied to all patients to whom $\mathrm{AD}$ are discontinued.

Controlled studies indicate no significant advantages of slow tapering compared to abrupt discontinuation as to onset of withdrawal symptoms $[2,3,17,39-41]$. Nonetheless, slow tapering with frequent contacts appears to be a reasonable strategy for many patients. There are, however, at least 2 exceptions. One occurs when switching from one $\mathrm{AD}$ to another due to lack or loss of efficacy: the value of tapering one $\mathrm{AD}$ and introducing the other during tapering or after discontinuation has not been established. We found that abrupt switching may be preferable, warning the patient about the intermediate phase when withdrawal symptoms of the first drug may occur and the benefits of the new one may not have appeared yet. A second exception may be given by the wish that some patients express to discontinue treatment abruptly in order to curtail the period in which withdrawal symptoms are experienced during tapering [28].

Reintroducing the $\mathrm{AD}$ that was used or switching from one $\mathrm{AD}$ to another (such as fluoxetine) to suppress clinical manifestations of withdrawal [27-31] are both highly questionable suggestions. A rational use of drugs depends on the balance of potential benefits and adverse effects applied to the individual patient [24]. One thing is reinstating an AD if relapse has occurred, another thing is to do it if withdrawal has ensued: we should be aware that, by doing this, we are simply postponing, and most likely aggravating, the problem. Tolerance does not necessarily develop to a specific drug, but it may occur as a reaction to particular effects of a drug, that may be shared by medications of the same class. In our model of tolerance [13, 15], the same opponent processes (most likely involving 5-HT1A autoreceptors) may be activated by different $\mathrm{AD}$. As a result, if we administer an $\mathrm{AD}$, regardless of whether it is the same or a different one, we may worsen the state of behavioral toxicity which is associated with withdrawal phenomena as well as other manifestations of oppositional tolerance [37]. We thus tend not to use AD treatment in case of withdrawal symptoms, even though we may be forced to use $\mathrm{AD}$, for the shortest possible time, for mood fluctuations in persistent postwithdrawal disorders which reach the intensity threshold of a major depressive disorder.

Even though tapering does not entail advantages as to the likelihood of withdrawal symptoms, it allows close monitoring of the clinical situation. Unfortunately we do not know, because there has not been adequate research, why certain patients develop withdrawal symptoms and others do not and whether these two groups differ from each other as to certain clinical and neurobiological characteristics. We usually tell patients that it is like flipping a coin: we have no way to know what is going to happen. With certain $\mathrm{AD}$ (paroxetine and venlafaxine) withdrawal symptoms are more likely, but we can never be sure. If withdrawal symptoms occur during tapering, this is a warning sign, even though we have observed patients who do not have problems during tapering, but only after the drug is actually discontinued. The red flag triggered by the appearance of withdrawal symptoms during tapering has taught us to adopt specific strategies, which involve both drug treatment and psychotherapeutic management. As to drug treatment, an important target could be to lessen withdrawal symptomatology by the use of psychotropic drugs that are not AD. Chouinard and Chouinard [2] have suggested the usefulness of antiepileptic drugs. In our clinical experience (but we lack pla- 
cebo-controlled studies), clonazepam at low doses, such as $0.5 \mathrm{mg}$ b.i.d., has emerged as the most preferable option. The choice of clonazepam is based on its antianxiety properties, paucity of side effects, facility to titrate, lack of significant interactions, mood-modulating effects, value in conjunction with or after $\mathrm{AD}$, and low likelihood for dependence compared to other BZD [42-45]. We thus add clonazepam to the $\mathrm{AD}$ we want to discontinue and, after 2 weeks of stabilization, we start tapering. Clonazepam may be increased when withdrawal symptomatology worsens. As withdrawal symptomatology may persist for months and build up into postwithdrawal disorders, also clonazepam treatment may need to be protracted.

A clinical issue that has attracted little attention is concerned with medical consequences of SSRI and SNRI discontinuation, since these $\mathrm{AD}$ may interact with a number of medical drugs [46, 47], such as anticoagulants, $\beta$-blockers, antihypertensive drugs and tamoxifen. Discontinuing AD may indeed require readjustment of medical therapies, which once again emphasizes the need for medical consultation, as we found to be the case in our unit. It is simply astonishing how these crucial clinical issues are ignored in guidelines and reviews that are supposed to inform clinical practice.

A further issue in discontinuing $\mathrm{AD}$ has to do with the frequent practice of polypharmacy in medicine [48] and psychiatry [49]. If we have a patient who is taking paroxetine, quetiapine and triazolam, how are we going to proceed? All three drugs are very likely to cause dependence and withdrawal symptoms. While it appears to be obvious that they cannot be discontinued all at the same time, there are unanswered clinical questions: Which drug should we stop first? What is the cross-tolerance of the drugs, that is how do they affect each other in terms of withdrawal symptomatology? Polypharmacy is simply not addressed by the literature on $\mathrm{AD}$ discontinuation, and yet it is a frequently encountered clinical reality. Assessing medication burden and polypharmacy [48] is thus another important factor in selecting discontinuation strategies.

\section{Psychotherapeutic Management}

A psychotherapeutic approach has been developed by the authors to accomplish AD discontinuation. The strategy encompasses 3 modules that may have variable duration according to the individualized needs of the patient. Patients are seen every 2 weeks for a number of sessions ranging from 16 to 24 . A preliminary version of this protocol has been used in persistent postwithdrawal disorders [12].

\section{First Module: Explanatory Therapy (4-6 Sessions)}

This psychotherapeutic approach was originally developed by Robert Kellner [50] for treating hypochondriasis and somatization, and was subsequently validated in a controlled investigation [51]. It consists in providing accurate information, teaching the principles of selective perception (attention to one part of the body makes the patient more aware of sensations in that part of the body than in other parts), reassurance, clarification, and repetition [50, 51]. We modified the protocol to manage withdrawal symptoms after $\mathrm{AD}$ discontinuation. It should be started before tapering $\mathrm{AD}$. In the first session, patients are encouraged to keep a diary in which they report the most disturbing symptoms that occur from one session to another. Accurate information is also provided. Patients are told that there is no way we can predict onset, severity, and duration of symptoms, that symptoms may start appearing during tapering and tend to have a peak 1 or 2 weeks after discontinuation. Withdrawal symptoms are new symptoms and may slowly subside within a month or may persist for a longer time (months). Development of the right attitude toward symptom perception (to be learned during psychotherapy) may hasten their disappearance.

Providing accurate information induces a "mental lighting" to patients. "If I had not been told that the symptoms I was experiencing after discontinuing venlafaxine were to be expected and that eventually there could be light at the end of the tunnel, I would have killed myself, because the mental pain was unbearable" wrote a young patient at the end of therapy.

The following sessions (which may range from a total of 3 to 5 , depending on the duration of tapering) deal with the patient's diary. The diary consists of a column where the patient describes the situation when the most distressing symptoms occur, another column with the symptoms that are experienced, and a third column where the patient formulates her thoughts and interpretations of the symptoms. The patient learns that not all symptoms that are experienced may be attributed to the AD (clarification). Table 1 provides an example of the diary of a schoolteacher during paroxetine tapering. The diary is important also when there are no immediate withdrawal symptoms upon tapering: symptoms may ensue with further tapering or after total discontinuation or may begin weeks or months after discontinuation (persistent postwithdrawal disorders). 
Table 1. Example of the AD tapering diary

\begin{tabular}{lll}
\hline Situation & Symptoms & Interpretation \\
\hline $\begin{array}{l}\text { I am at home trying to } \\
\text { prepare my lesson for } \\
\text { the class }\end{array}$ & $\begin{array}{l}\text { I am feeling bad, confused and not } \\
\text { enough prepared; I am having electric } \\
\text { shock sensations; my lesson will be a fail- } \\
\text { ure, and students will be disappointed }\end{array}$ & $\begin{array}{l}\text { Electric shock sensations are caused } \\
\text { by lowering the dose of the drug; I } \\
\text { had the other symptoms also before } \\
\text { starting the medication }\end{array}$ \\
\hline
\end{tabular}

Patients are encouraged to keep on doing life activities and to pay as little attention as possible to symptoms. There are days which are bad, but, if patients try to react, the days get better. In patients with a passive attitude to life and plenty of opportunities for paying attention to their symptoms, activity scheduling should be introduced. Repeated reassurance is also another important therapeutic ingredient of explanatory therapy (e.g." "it is quite frequent to have these symptoms upon tapering or after discontinuation of this drug," "it is not a relapse, but a withdrawal reaction;" "it is just a transient phase, soon it may be over").

Some motivational elements may be introduced explaining the concept of behavioral toxicity [37]. Patients are reminded that the drug had become toxic to them and that in the long run they will feel much better. If clinical phenomena related to oppositional tolerance (e.g., loss of efficacy, paradoxical effects) or major side effects (e.g., weight gain) occurred, they may be used effectively to suggest the importance of getting the $\mathrm{AD}$ off the body and the potential harms of simply changing the type of $\mathrm{AD}$. Finally, some lifestyle suggestions are given (avoidance of alcohol and limited caffeine consumption, physical exercise, sleep hygiene, balanced diet). The structure of this first module is summarized in Table 2. Ideally this module should be monitored by the treating psychiatrist alone or jointly with the psychologist. The medical consultant may also be involved.

\section{Second Module: Reassessment and Cognitive Behavior Treatment (6-10 Sessions)}

Four weeks after AD discontinuation, as customary in the sequential treatment schema [26,32], it is important to reassess the patient. The psychiatric interview should be particularly focused on the symptomatology that occurred before $\mathrm{AD}$ treatment, whether depression, anxiety or both. Clinimetric tools such as the Clinical Interview for Depression [52] may be very helpful in detecting subclinical symptoms. Other indices may tap withdrawal symptomatology $[34,35]$. Examination of the diary (self-
Table 2. Goals of the first module

1 Checking the general status of the patient

2 Review of the diary, with particular reference to the onset of withdrawal symptoms

3 Encouraging the patient to keep on tapering and eventually discontinuing the antidepressant

4 Providing accurate information, teaching the principles of selective perception, reassurance, clarification, and repetition

5 Activity scheduling if necessary

6 Homework assignments

7 Lifestyle suggestions

monitoring) could yield other important insights. A new macroanalysis should be performed.

An important clinical question is whether the patient received any effective, evidence-based psychotherapeutic treatment in addition to pharmacotherapy. If yes, which was exactly the protocol? Was psychotherapy performed while taking $\mathrm{AD}$ ? Pharmacological treatment may decrease the efficacy of the psychotherapeutic approach (patients report that, particularly with paroxetine and venlafaxine, they felt themselves "anesthetized" and "detached") [37].

The strategy of this module may be quite different in mood and anxiety disorders. In depression there is the need for addressing residual symptomatology and cognitive lifestyle that may, in the long run, lead to relapse [4, 5]. In anxiety disorders, there is often the need for treating the original symptomatology that was only temporarily managed by pharmacological treatment. We use cognitive behavior strategies (CBT), encompassing both cognitive restructuring and homework exposure, according to standard methods [53-55]. However, monitoring of withdrawal symptoms that may persist has to be maintained during the course of this second module. The psychotherapist should balance the treatment of the original symptoms and the occurrence of new symptoms according to the patient's priorities and needs. 
Table 3. Goals of the second module

1 Checking the general status of the patient

2 Review of the diary, with particular reference to both the trajectory of withdrawal symptomatology and affective symptoms

3 Providing information, reassurance, clarification, and repetition

4 Cognitive restructuring

5 Activity scheduling

6 Homework assignments

7 Lifestyle suggestions
The structure of the second module is summarized in Table 3. It can be accomplished by the psychologist, but a couple of assessments by the treating psychiatrist are essential. The medical consultant may be needed again. In our Affective Disorders Program, the internist evaluates also the level of stress and allostatic load using the PsychoSocial Index $[56,57]$.

\section{Third Module: Well-Being Therapy (6-8 Sessions)}

Well-being therapy (WBT) is a short-term psychotherapeutic strategy that emphasizes self-observation of psychological well-being, with the use of a structured diary, cognitive restructuring of interfering thoughts and/ or behaviors through cognitive behavioral techniques, and homework assignments (i.e., pursuing optimal experiences) $[58,59]$. WBT has been validated in a number of randomized controlled trials. It has been used mainly in sequential combination after CBT $[58,59]$. In our clinical experience, WBT has emerged as a crucial treatment component for a variety of reasons.

Many patients are convinced that they cannot make it without $\mathrm{AD}$, because they are weak and vulnerable [58]. The difficulties in discontinuing the AD may lead to impairments in various dimensions of psychological wellbeing: environmental mastery ("I cannot function without antidepressants"); autonomy ("I am ill. I cannot do anything by myself"); purpose in life ("There is no life after antidepressants"); positive relations with others ("Without antidepressants I am insufferable, because of my irritability and mood swings"); personal growth ("Things will simply get worse and worse").

Use of the well-being diary, associated with cognitive restructuring and exposure homework, may provide a different outlook to patients. Table 4 is taken from the diary of the same schoolteacher as in Table 1. Flourishing and resilience can be promoted leading to a positive eval- uation of one's self, a sense of continuous growth and development, the belief that life is purposeful and meaningful, quality relations with others, the capacity to manage affectively one's life, and a sense of self-determination. A decreased vulnerability to depression and anxiety has been demonstrated after WBT in mood and anxiety disorders, and this may entail a lower likelihood to use $\mathrm{AD}$ in the future $[58,59]$.

The structure of the third module is described in Table 5. As was found to be the case in the second module, the psychological work needs to be associated with psychiatric and medical follow-ups.

\section{On Miseducation}

Twenty years ago, this journal anticipated the current medical scenario dominated by corporate interests which result in self-selected academic oligarchies (special interest groups) that influence clinical and scientific information [60]. Members of special interest groups, by virtue of their financial power and close ties among themselves, have the task of systematically preventing dissemination of data which may be in conflict with their interests [61]. Corporate powers fused with academic medicine to create an unhealthy alliance that works against objective reporting of clinical research, sets up meetings and symposia with the specific purpose of selling the participants to sponsors, and substantially controls journals, medical associations, and related foundations (through direct support and/or advertising). The growth of evidence-based medicine, with its emphasis on systematic reviews and guidelines, provided an ideal ground for multiplying the effects of financial conflicts of interest in medicine [62].

In the early nineties, when the reports on withdrawal syndromes upon discontinuing SSRI began to appear [7], it was crucial to downplay such occurrences. At that time, the commercial plan was to extend the use of SSRI to other psychiatric disorders (particularly anxiety disturbances) and to prolong their administration to the longest possible time. Awareness of the occurrence of dependence would have run counter to such a strategy. A concerted action became operational:

(a) Withdrawal reactions were promptly renamed as discontinuation syndromes, as if they were different from what was known about other psychotropic drugs, such as BZD. There was no evidence to support that dependence was different with SSRI $[2,3,63]$ and SNRI $[2,17]$. Indeed, in our clinical experience it is far more difficult to discontinue a drug such as paroxetine or venlafaxine than 
Table 4. Example of the well-being diary

\begin{tabular}{llll}
\hline Situation & Well-being & $\begin{array}{l}\text { Interrupting thoughts or behav- } \\
\text { ior }\end{array}$ & Observer \\
\hline $\begin{array}{l}\text { In class I was able to handle } \\
\text { some disturbing students }\end{array}$ & $\begin{array}{l}\text { It is the first time I am able to } \\
\text { accomplish this; I am feeling } \\
\text { very good }\end{array}$ & $\begin{array}{l}\text { It is just a single episode; } \\
\text { students will keep on ignoring } \\
\text { me as usual }\end{array}$ & $\begin{array}{l}\text { It is not a single episode; I am } \\
\text { getting more control on myself } \\
\text { and on people around me }\end{array}$ \\
\hline
\end{tabular}

Table 5. Goals of the third module

1 Checking the general status of the patient and the potential development of a persistent postwithdrawal disorder

2 Introducing the change in focus of psychotherapy (wellbeing instead of distress)

3 Giving homework assignments (well-being diary): monitoring of instances of well-being, thoughts and/or behaviors leading to premature interruption, with the introduction of the observer's column (cognitive restructuring)

4 Introducing the concept of optimal experiences and their pursuit

5 Activity scheduling

6 Homework assignments

7 Lifestyle suggestions

any BZD. Both physicians and patients were taught that the problem could have appeared only with abrupt discontinuation of $\mathrm{AD}$ and that, if symptoms arose, they had to be considered signs of relapse, with prompt readministration of the $\mathrm{AD}$.

(b) BZD, because of their widespread use and their limited cost, were a major obstacle to the introduction of the new $\mathrm{AD}$ in anxiety disorders. In anxiety disorders, when directly compared to $\mathrm{AD}, \mathrm{BZD}$ were found to be more or as effective and with fewer side effects [64]. In anxious and/or minor depression BZD were a valid therapeutic option [65]. A commercial war was thus started: the dependence potential of BZD was dramatized, and their prescription was hindered in all possible ways, despite the clinical value of this class of medication $[66,67]$. Physicians thus learned that BZD were bad and could cause dependence, whereas AD were devoid of such effects. This was probably the most spectacular achievement of propaganda in psychiatry.

(c) Prolongation of pharmacological treatment with the claim to maintain the clinical responses obtained in the short term was another commercial target for SSRI and SNRI. The evidence supporting this strategy was mainly based on clinical trials where remitted patients were randomized to drug continuation or placebo, without any differentiation between withdrawal syndromes and relapse [68]. Thus, in the group that underwent drug tapering and discontinuation, we have no way to know how many of the relapses were actually withdrawal and postwithdrawal syndromes [24]. As a result, the efficacy of $\mathrm{AD}$ in preventing relapse in depression compared to placebo relies on hiding the existence of withdrawal syndromes that are misinterpreted as new illness episodes.

(d) Special interest groups operating in funding agencies and medical journals made sure that iatrogenic components of depression could not get attention and funding, even though these phenomena may explain a large part of clinical outcomes, such as chronicity. An important research question arises with withdrawal phenomena related to SSRI and SNRI: are these distressing but selflimiting reactions or are they signs of something else? They may be manifestations of behavioral toxicity (the oppositional model of tolerance), potentially related to loss of clinical effects, increased vulnerability to relapse, resistance when treatment is reinstituted, unresponsiveness, paradoxical effects [24].

A major consequence of these commercial strategies has been that patients experiencing anguish and mental pain of withdrawal syndromes have not received appropriate medical attention and have been forced to refer themselves to websites, groups, associations, which had the recognized merit of providing support but could not offer the medical competence that was required, particularly as to medical comorbidities. Professional organizations and scientific societies have major responsibilities in not assuring proper care to people in need of it.

\section{Conclusions}

The damages of miseducation are difficult to overcome. AD are important and potentially life-saving drugs if the proper indications are endorsed. However, the pre- 
scribing physician is currently driven by an overestimated consideration of potential benefits, with little attention to the likelihood of responsiveness and neglect of potential vulnerability to the adverse effects of treatment $[24,69]$.

A rational use of $\mathrm{AD}$ that incorporates all potential benefits and harms consists in targeting their application only to the most persistent cases of depression, limiting their use to the shortest possible time, and reducing their utilization in anxiety disorders (unless a major depressive disorder is present or other treatments have been ineffective). Since behavioral toxicity appears to be related to the dosages of $\mathrm{AD}$, the lowest dose of these agents that seems to be both effective and well tolerated should be employed [70]. Augmenting strategies (i.e., adding new psychotropic drugs to the regimen) need to be carefully weighed, if not avoided, because of their strong link with behavioral toxicity [24]. One should be particularly concerned with young patients who are given $\mathrm{AD}$ for anxiety disorders and prolong this treatment indefinitely without undergoing any form of psychotherapy. What will be the longterm outcome of their disturbances? Will tolerance develop and trigger deterioration and refractoriness?

The time has come to initiate research on withdrawal phenomena related to $\mathrm{AD}$, and to redefine the use and indications of these medications, including their differential likelihood of inducing behavioral toxicity [24].

\section{Acknowledgments}

The authors are indebted to the members of the Affective Disorders Program where these observations originated: Nicoletta Sonino (consultant for internal medicine and endocrinology), Chiara Rafanelli and Anna Rita Raffi (consultants for psychiatry and psychotherapy), Jenny Guidi, Laura Staccini, and Elena Tomba (consultants for psychology and psychotherapy). They are also grateful to Giada Benasi, Guy Chouinard, Fiammetta Cosci, and Emanuela Offidani for providing important sources of insight.

\section{References}

1 Scholten WD, Batelaan NM, van Oppen P, Smit JH, Hoogendoorn AW, van Megen HJGM, Cath DC, van Balkom AJLM: The efficacy of a group CBT relapse prevention program for remitted anxiety disorder patients who discontinue antidepressant medication: a randomized controlled trial. Psychother Psychosom 2018;87:240-242.

2 Chouinard G, Chouinard VA: New classification of selective serotonin reuptake inhibitor withdrawal. Psychother Psychosom 2015;84: 63-71.

3 Fava GA, Gatti A, Belaise C, Guidi J, Offidani E: Withdrawal symptoms after selective serotonin reuptake inhibitor discontinuation: a systematic review. Psychother Psychosom 2015;84:72-81.

4 Fava GA, Grandi S, Zielezny M, Canestrari R, Morphy MA: Cognitive behavioral treatment of residual symptoms in primary major depressive disorder. Am J Psychiatry 1994;151: 1295-1299.

5 Fava GA, Rafanelli C, Grandi S, Conti S, Belluardo P: Prevention of recurrent depression with cognitive behavioral therapy. Arch Gen Psychiatry 1998;55:816-820.

6 Fava GA, Grandi S, Belluardo P, Savron G, Raffi AR, Conti S, Saviotti FM: Benzodiazepines and anxiety sensitivity in panic disorder. Prog Neuropsychopharmacol Biol Psychiatry 1994;18:1163-1168.

7 Fava GA, Grandi S: Withdrawal syndromes after paroxetine and sertraline discontinuation. J Clin Psychopharmacol 1995;15:374375 .
8 Fava GA, Bernardi M, Tomba E, Rafanelli C: Effects of gradual discontinuation of selective serotonin reuptake inhibitors in panic disorder with agoraphobia. Int J Neuropsychopharmacol 2007;10:835-838.

9 Fava GA, Rafanelli C, Grandi S, Conti S, Ruini C, Mangelli L, Belluardo P: Long-term outcome of panic disorder with agoraphobia treated by exposure. Psychol Med 2001;31: 891-898.

10 Rickels K, Schweizer E, Case G, Greenblatt DJ: Long-term therapeutic use of benzodiazepines. I. Effects of abrupt discontinuation. Arch Gen Psychiatry 1990;47:899-907.

11 Belaise C, Gatti A, Chouinard VA, Chouinard G: Patient online report of selective serotonin reuptake inhibitor (SSRI) induced persistent postwithdrawal anxiety and mood disorders. Psychother Psychosom 2012;81:386-388.

12 Belaise C, Gatti A, Chouinard VA, Chouinard G: Persistent postwithdrawal disorders induced by paroxetine, a selective serotonin reuptake inhibitor, and treated with specific cognitive behavioral therapy. Psychother Psychosom 2014;83:247-248.

13 Fava GA: Can long-term treatment with antidepressant drugs worsen the course of depression? J Clin Psychiatry 2003;64:123-133.

14 Fava GA, Mangelli L: Mania associated with venlafaxine discontinuation. Int J Neuropsychopharmacol 2003;6:89-90.

15 Fava GA, Offidani E: The mechanisms of tolerance in antidepressant action. Prog Neuropsychopharmacol Biol Psychiatry 2011;35: 1593-1602.
16 Bosman RC, Waumans RC, Jacobs GE, Voshaar O, Muntingh ADT, Batelaan NM, van Balkom AJLM: A failure to respond after reinstating antidepressant medication. Psychother Psychosom 2018, Epub ahead of print.

17 Fava GA, Benasi G, Lucente M, Offidani E, Cosci F, Guidi J: Withdrawal symptoms after serotonin-noradrenaline reuptake inhibitors discontinuation. Psychother Psychosom 2018;87:195-203.

18 Fava GA, Park SK, Dubovsky SL: The mental health clinic: a new model. World Psychiatry 2008;7:177-181.

19 Carvalho AF, Sharma MS, Brunoni AR, Vieta E, Fava GA: The safety, tolerability and risks associated with the use of newer generation antidepressant drugs. Psychother Psychosom 2016;85:270-288.

20 Maslej MM, Bolker BM, Russell MJ, Eaton K, Durisko Z, Hollon SD, Swanson GM, Thomson JA, Mulsant BH, Andrews PW: The mortality and myocardial effects of antidepressants are moderated by pre-existing cardiovascular disease. Psychother Psychosom 2017;86:268-282.

21 Grace SL, Medina-Inojosa JR, Thomas RJ, Krause H, Vickers-Douglas KS, Palmer BA, Lopez-Jimenez F: Antidepressant use by class: association with major adverse events in patients with coronary artery disease. Psychother Psychosom 2018;87:85-94. 
22 Huang WS, Bai YM, Hsu JW, Huang KL, Tsai CF, Su TP, Li CT, Lin WC, Tsai SJ, Pan TL, Chen TJ, Chen MH: Use of proton pump inhibitors and risk of major depressive disorder. Psychother Psychosom 2018;87:62-64.

23 Fux M, Taub M, Zohar J: Emergence of depressive symptoms during treatment for panic disorder with specific 5-hydroxytryptophan reuptake inhibitors. Acta Psychiatr Scand 1993;88:235-237.

24 Fava GA: Rational use of antidepressant drugs. Psychother Psychosom 2014;83:197204.

25 Huijbregts KM, Hoogendoorn AW, Slottje P, van Balkom AJLM, Batelaan NM: Long-term and short-term antidepressant use in general practice. Psychother Psychosom 2017;86: 362-369.

26 Guidi J, Tomba E, Fava GA: The sequential integration of pharmacotherapy and psychotherapy in the treatment of major depressive disorder: a meta-analysis of the sequential model and a critical review of the literature. Am J Psychiatry 2016;173:128-137.

27 Lejoyeux M, Ades J: Antidepressant discontinuation. J Clin Psychiatry 1997;58(suppl 7): 11-16.

28 Haddad PM: Antidepressant discontinuation syndromes. Drug Safety 2001;24:183-197.

29 Schatzberg AF, Blier P, Delgado PL, Fava M, Haddad PM, Shelton RC: Antidepressant discontinuation syndrome. J Clin Psychiatry 2006;67(suppl 4):27-30.

30 Warner CH, Bobo W, Warner C, Reid S, Rachal J: Antidepressant discontinuation syndrome. Am Fam Physician 2006;74:449-456.

31 Wilson E, Lader M: A review of the management of antidepressant discontinuation symptoms. Ther Adv Psychopharmacol 2015; 5:357-358.

32 Tomba E, Fava GA: Treatment selection in depression: the role of clinical judgment. Psychiatr Clin North Am 2012;35:87-98.

33 Fava GA, Tomba E: Treatment of comorbid anxiety disorders and depression; in Emmelkamp P, Ehring T (eds): The Wiley Handbook of Anxiety Disorders. Chichester, Wiley, 2014, pp 1165-1182.

34 Rosenbaum JF, Fava M, Hoog SL, Ascroft C, Krebs WB: Selective serotonin reuptake inhibitor discontinuation syndrome: a randomized clinical trial. Biol Psychiatry 1998;44:7787.

35 Cosci F, Chouinard G, Chouinard V-A, Fava GA: The Diagnostic Clinical Interview for Drug Withdrawal 1 (DID-W1) - new symptoms of selective serotonin reuptake inhibitors (SSRI) or serotonin noradrenaline reuptake inhibitors (SNRI): inter-rater reliability. Riv Psichiatr 2018;53:95-99.

36 Chouinard G, Samaha AN, Chouinard V-A, Peretti C-S, Kanahara N, Takase M, Iyo M: Antipsychotic-induced dopamine supersensitivity psychosis. Psychother Psychosom 2017;86:189-219.
37 Fava GA, Cosci F, Offidani E, Guidi J: Behavioral toxicity revisited: iatrogenic comorbidity in psychiatric evaluation and treatment. J Clin Psychopharmacol 2016;36:550-553.

38 Bainum TB, Fike DS, Mechelay D, Haase K: Effect of abrupt discontinuation of antidepressants in critically ill hospitalized patients. Pharmacotherapy 2017;37:1231-1240.

39 Tint A, Haddad PM, Anderson IM: The effect of rate of antidepressant tapering on the incidence of discontinuation symptoms: a randomised study. J Psychopharmacol 2008;22: 330-332.

40 Gallagher J, Strzinek RA, Cheng RJ, Ausmanas MK, Astl D, Seljan P: The effect of dose titration and dose tapering on the tolerability of desvenlafaxine in women with vasomotor symptoms associated with menopause. J Womens Health 2012;21:188-198.

41 Khan A, Musgnung J, Ramey T, Messig M, Buckley G, Ninan P: Abrupt discontinuation compared with a 1-week taper regimen in depressed outpatients treated for 24 weeks with desvenlafaxine $50 \mathrm{mg} / \mathrm{d}$. J Clin Psychopharmacol 2014;34:365-368

42 Chouinard G: Issues in the clinical use of benzodiazepines: potency, withdrawal and rebound. J Clin Psychiatry 2004;65(suppl 5):712.

43 Pollack MH, Van Ameringen M, Simon N, Worthinton JW, Hoge EA, Keshaviah A, Stein MB: A double-blind randomized controlled trial of augmentation and switch strategies for refractory social anxiety disorder. Am J Psychiatry 2014;171:44-53.

44 Cloos JM, Bocquet V, Rolland-Portal I, Koch P, Chouinard G: Hypnotics and triazolobenzodiazepines - best predictors of high-dose benzodiazepine use. Psychother Psychosom 2015;84:273-283.

45 Nardi AE, Cosci F, Balon R, Weintraub SJ, Freire RC, Krystal JH, Roth T, Silberman EK, Sonino N, Fava GA, Starcevic V, Dubovsky SL, Salzman C, Rickels K, Greenblatt DJ, Shader RI, Chouinard G: The prescription of benzodiazepines for panic disorder. J Clin Psychopharmacol 2018;38:283-285.

46 Fava GA, Sonino N: Depression associated with medical illness. CNS Drugs 1996;5:175189.

47 Spina E, Trifirò G, Caraci F: Clinically significant drug interactions with newer antidepressants. CNS Drugs 2012;26:39-67.

48 Gnjidic D, Tinetti M, Allore HG: Assessing medication burden and polypharmacy: finding the perfect measure. Exp Rev Clin Pharmacol 2017; 10:345-347.

49 Ghaemi SN (ed): Polypharmacy in Psychiatry. New York, Dekker, 2002.

50 Kellner R: Psychotherapeutic strategies in the treatment of psychophysiological disorders. Psychother Psychosom 1979;32:91-100.

51 Fava GA, Grandi S, Rafanelli C, Fabbri S, Cazzaro M: Explanatory therapy in hypochondriasis. J Clin Psychiatry 2000;61:317-322.
52 Guidi J, Fava GA, Bech P, Paykel E: The Clinical Interview for Depression. Psychother Psychosom 2011;80:10-27.

53 Beck AT, Rush AJ, Shaw BF, Emery G: Cognitive Therapy of Depression. New York, Guilford, 1979.

54 Marks IM: Fears, Phobias and Rituals. New York, Oxford University Press, 1987.

55 Clark DA, Beck AT: Cognitive Therapy of Anxiety Disorders. Science and Practice. New York, Guilford, 2010.

56 Sonino N, Fava GA: A simple instrument for assessing stress in clinical practice. Postgrad Med J 1998;74:408-410.

57 Piolanti A, Offidani E, Guidi J, Gostoli S, Fava GA, Sonino N: Use of the PsychoSocial Index: a sensitive tool in research and practice. Psychother Psychosom 2016;85:337-345.

58 Fava GA: Well-Being Therapy. Treatment Manual and Clinical Applications. Basel, Karger, 2016.

59 Fava GA: Well-being therapy: current indications and emerging perspectives. Psychother Psychosom 2016;85:136-145.

60 Fava GA: All our dreams are sold. Psychother Psychosom 1998;67:191-193.

61 Fava GA: The hidden costs of financial conflicts of interest in medicine. Psychother Psychosom 2016;85:65-70.

62 Fava GA: Evidence-based medicine was bound to fail. J Clin Epidemiol 2017;84:3-7.

63 Nielsen M, Hansen EH, Goztsche PC: What is the difference between dependence and withdrawal reactions? A comparison of benzodiazepines and selective serotonin re-uptake inhibitors. Addiction 2012;107:900-908.

64 Offidani E, Guidi J, Tomba E, Fava GA: Efficacy and tolerability of benzodiazepines versus antidepressants in anxiety disorders. Psychother Psychosom 2013;82:355-362.

65 Benasi G, Guidi J, Offidani E, Balon R, Rickels $\mathrm{K}$, Fava GA: Benzodiazepines as a monotherapy in depressive disorders: a systematic review. Psychother Psychosom 2018;87:65-74.

66 Balon R, Chouinard G, Cosci F, Dubovsky SL, Fava GA, Freire RC, Greenblatt DJ, Krystal JH, Nardi AE, Rickels K, Roth T, Salzman C, Shader R, Silberman EK, Sonino N, Starcevic V, Weintraub SJ: International Task Force on Benzodiazepines. Psychother Psychosom 2018;87:193-194.

67 Jorgensen MB, Osler M: Should benzodiazepines be avoided? Acta Psychiatr Scand 2018; 138:89-90.

68 Geddes JR, McCarney SM, Davies C, Furukawa TA, Kupfer DJ, Frank E, Goodwin GM: Relapse prevention with antidepressant drug treatment in depressive disorders: a systematic review. Lancet 2003;361:653-661.

69 Tomba E, Guidi J, Fava GA: What psychologists need to know about psychotropic medications. Clin Psychol Psychother 2018;25: 181-187.

70 Baldessarini RJ, Ghaemi SN, Viguera AC: Tolerance in antidepressant treatment. Psychother Psychosom 2002;71:177-179. 